\title{
Calidad industrial de variedades de trigo, Itapúa 80, Itapúa 85 y Canindé 21
}

\author{
Industrial quality of Itapúa 80, Itapúa 85 and Canindé 21 wheat varieties \\ Graciela Cabrera Arredondo ${ }^{*}$, Man Mohan Kohli², Mabel Agüero ${ }^{3}$, Epifanio Altamirano ${ }^{3}$ y \\ Gustavo Benítez Quintana ${ }^{4}$ \\ ${ }^{1}$ Instituto Paraguayo de Tecnología Agraria. IPTA/CIHB, km 48,5, Caacupé, Paraguay. \\ 2 Cámara Paraguaya de Exportadores y Comercializadores de Cereales y Oleaginosas, CAPECO, Avda. Brasilia 840, \\ Asunción, Paraguay. \\ ${ }^{3}$ Instituto Paraguayo de Tecnología Agraria. IPTA/CICM, km 16 Ruta 6, Capitán Miranda, Paraguay. \\ ${ }^{4}$ Cooperativa Colonias Unidas, CCU. Avda. Gas par Rodríguez de Francia, Obligado, Paraguay. \\ *Autor para correspondencia (gc580613@hotmail.com) \\ Recibido: 30/06/2016; Aceptado: 19/08/2016.
}

10.18004/investig.agrar.2016.dic ie mbre .95-100

\section{RESUMEN}

En el marco del Programa Nacional de Trigo, dentro del convenio IPTA/CAPECO/INBIO se busca identificar nuevas variedades con buenas características agronómicas, tolerancia y/o resistencia a enfermedades y buena calidad industrial para distintas zonas productivas del país. Fueron lanzadas al mercado nacional tres variedades, ITAPUA 80, ITAPUA 85 y CANINDÉ 21, durante 2013/15, evaluándose su calidad industrial a través de análisis físico químico y reológico. En cuanto a los caracteres físicos, las tres variedades se encuentran dentro de los rangos aceptados por la industria, teniendo los valores de peso hectolítrico $(\mathrm{PH})$ entre 76 y $79 \mathrm{~kg} \mathrm{hL}^{-1}$ y los valores de peso de mil granos (PMG) entre 29,85 y $33,85 \mathrm{~g}$. El análisis de las características de la calidad industrial fue medido por las pruebas de proteína, cantidad y calidad del gluten, valores del índice de caída o 'falling number' y la alveografía. Todos los análisis demostraron la excelente calidad panadera de las tres variedades agrupándolas en aptas para los propósitos de la industria tanto nacional como internacional. Entre las tres variedades, Canindé 21 fue considerado como el de mejor gluten por sus propiedades de resistencia al amasado y puede ser proveído a la industria con precios diferenciados.

Palabras clave: Triticum aestivum L, variedades, calidad industrial.

\begin{abstract}
The objective of the National Wheat Program, IPTA / CAPECO / INBIO is to identify new varieties with good agronomic characteristics, tolerance and / or resistance to disease and good industrial quality for the country's different production areas. Three new varieties, ITAPUA 80, ITAPUA 85 and Canindé 21, released during 2013/15, were evaluated for their industrial quality through chemical and physical analysis including rheological properties of dough. Regarding their physical characteristics, the three varieties are within the ranges accepted by the industry, with hectoliter weight $(\mathrm{PH})$ values between 76 and $79 \mathrm{~kg} / \mathrm{hl}$ and Thousand Kernel Weight (TKW) values between 29.85 and $33.85 \mathrm{gs}$. Industrial quality was measured by protein assay, gluten quantity and quality, Falling Number index value and Alveography. The analyzes showed the excellent bakery quality of all three varieties, which were all deemed suitable for the industry both nationally and internationally. Among the three varieties, Canindé 21 was considered as the one with the best gluten for its properties of kneading resistance strength, which made it eligible to be supplied to the industry at premium prices. Key words: Triticum aestivum $L$, varieties, industrial quality.
\end{abstract}




\section{INTRODUCCIÓN}

La calidad del grano de una variedad de trigo está determinada, principalmente, por características genéticas, pero también está muy influenciada por factores ambientales y por la interacción que pueda existir entre la variedad y el ambiente en el cual se desarrolla (Matus 2010). Es por eso que los desafíos para el mejoramiento genético incluyen la necesidad de crear variedades adaptadas, alto potencial de rendimiento, mayor tolerancia a las enfermedades y una elevada calidad industrial.

Otro factor fundamental para asegurar la obtención de un grano de buena calidad está asociado al manejo agronómico del cultivo por los productores (Matus y Vega 2004). La variedad mejorada de trigo es un componente fundamental para lograr el éxito en la producción, ya que ha sido desarrollada para alcanzar un comportamiento óptimo en cuanto a rendimiento, calidad industrial y tolerancia o resistencia a enfermedades.

También, Williams et al. (2008) y De la O et al. (2010) sugirieron que las variedades y el ambiente son factores que afectan directamente a la expresión de la calidad. Es por esto que una variedad de trigo debe reunir un conjunto de características deseables desde el punto de vista de productividad, es decir, que tenga un alto rendimiento, que al final se traduce en el principal factor de rentabilidad para el agricultor, una variedad debe ser capaz de producir un grano de calidad, lo cual es fundamental para su comercialización (Matus y Vega 2004).

Para el mejorador genético, el concepto de estabilidad en la calidad o respuesta al ambiente representa los cambios del genotipo a través de ambientes diferentes que afectan una eficiente selección. Para los molineros y panaderos la consistencia en la calidad de las variedades es muy importante, ya que no requiere de cambios en los procesos (Cuniberti et al.2004).

Existen varias características de calidad que son importantes a considerar al momento de decidir qué variedad sembrar, las cuales también son tomadas en cuenta por la industria molinera durante la compra del grano, ya que están directamente relacionadas con la obtención de una harina de buena calidad.

En el mundo, la demanda de trigo es cada vez más selectiva, requiriendo trigos específicos para determinados usos, adaptando las nuevas tecnologías y s u marketing en función de las demandas cambiantes (Cuniberti et al. 2004).

Como resultados de las investigaciones realizadas a través del Convenio IPTA- CAPECO-INBIO, tres variedades de trigo, Itapúa 80, Itapuá 85 y Canindé 21, fueron lanzadas durante el periodo de 2013-2014.

El objetivo de este trabajo fue determinar la calidad industrial de las nuevas variedades, a través de los análisis físico-químico y reológico de sus granos y harinas.

\section{MATERIALES Y MÉTODOS}

Las muestras de los granos para el análisis de calidad se obtuvieron de los ensayos de rendimiento del Programa de Investigación de Trigo, de tres localidades: Capitán Miranda (CICM), Colonia Yguazú (CETAPAR) e Yhovy (CEY) y de tres ciclos de cultivo: 2012, 2013 y 2014. Las variedades analizadas fueron Itapuá 80, Itapuá 85 y Canindé 21. Para cada variedad se preparó y analizó una muestra por año a partir de la mezcla homogeneizada de los granos de tres repeticiones de los ensayos. Finalmente, se calculó la media de los resultados por localidad de los tres años considerados.

Los análisis físicos-químicos de las variedades fueron realizados en el laboratorio del Centro de Investigación Capitán Miranda (CICM), del Instituto Paraguayo de Tecnología Agraria (IPTA) y el análisis reológico en el laboratorio de la Cooperativa Colonias Unidas.

Los métodos de análisis de calidad aplicados fueron:

En los granos: El Peso Hectolítrico $\left(k g h L^{-1}\right)$ se determinó con una balanza volumétrica de $500 \mathrm{~mL}$ (OHAUS), de $52 \mathrm{~mm}$ de caída de acuerdo a la técnica estandarizada de la AACC 55-10 (American Association of Cereal Chemists 2000). Para el peso de 1.000 granos (g), se utilizó un contador electrónico de granos, en donde los 1.000 granos contados se pesaron y de acuerdo al método IRAM 15853 (Instituto Argentino de Normalización y Certificación) se obtuvo el peso de 1.000 granos. El porcentaje de proteína se determinó con el analizador de granos, utilizando tecnología NIRT de la FOSS con el equipo Infratec 1241 según el método 39-00 de la AACC, 2000.

En las harinas: Para la determinación del Índice de caída o 'Falling Number' (s) se utilizó el equipo automático 
'Falling Number' de Perten Suecia, modelo 1400 de acuerdo al método de Harberg-Perten estandarizado por la AACC, 2000 (56-81). El porcentaje de Gluten Húmedo se determinó por medio del equipo Glutomatic marca Falling Number, modelo 2100 (Huddinge, Suecia), por el método 38-11 de la AACC, 2000 y la determinación de Alveografía fueron determinadas por un Alveógrafo de Chopin (Trippette \& Renaud, Paris, Francia) en una muestra de $250 \mathrm{~g}$ de acuerdo con el método 54-30 de la AACC, 2000.

La interpretación de los resultados se realizó según la clasificación de calidad de acuerdo a Williams et al. (1988).

\section{RESULTADOS Y DISCUSIÓN}

El peso hectolítrico (PH) es una medida de la homogeneidad de la partida de trigo, factor clave en el proceso industrial. Los molineros toman al $\mathrm{PH}$ como característica importante, por su relación con el porcentaje de extracción de harina para productos panificables, y se utiliza generalmente a nivel mundial en la comercialización del trigo para determinar su precio y uso industrial (Vázquez et al. 2001). Por consiguiente, Vázquez (2006) considera al PH como una buena estimación tanto de la calidad física del grano, como de la calidad molinera. A nivel comercial, alcanzar valores de PH por encima de 76 representa una bonificación en el precio recibido por el productor ya que produce un mayor porcentaje de harina en la molienda. Otros factores como la presencia de granos inmaduros, trigos dañados severamente por sequía o por enfermedades disminuyen el peso hectolítrico y por lo tanto el rendimiento harinero (Halverson y Zeleny 1988; Gaines et al. 1997, Peña et al. 2008).

Las variedades bajo estudio, Itapúa 80, Itapúa 85 y Canindé 21, presentaron valores excelentes de $\mathrm{PH}$, entre $76 \mathrm{~kg} \mathrm{hL}^{-1}$ y $79 \mathrm{~kg} \mathrm{hL}^{1}$, en las tres localidades y años considerados. Estos valores son considerados como granos pesados según la clasificación de calidad que permite un alto porcentaje de extracción de harina.

El peso de 1000 granos, es un indicador de calidad de las harinas y esto se basa en que los granos grandes y den s os contienen mayor proporción de endospermo y por lo tanto mayor rendimiento de harina (Palmer 1989; Wilson y Godiño 2000; Vázquez 2009). Esta característica se encuentra altamente influenciada por el clima, especialmente la temperatura, durante el periodo de llenado de grano. De acuerdo a la clasificación del peso de grano, las tres variedades se encuentran en un rango de grano pequeño a mediano ( $26 \mathrm{~g}$ a $35 \mathrm{~g}$ ). Las variaciones de las temperaturas entre años y localidades son responsables por las fluctuaciones de $29,85 \mathrm{~g}$ a $33,85 \mathrm{~g}$ observadas entre las variedades Itapúa 80, Itapuá 85 y Canindé 21 (Tabla 1). El tamaño más requerido por la industria nacional molinera es el mediano, porque granos grandes provocan problemas en la limpieza y los granos pequeños atraviesan los tamices de limpieza y ocasionan pérdida por la disminución de la cantidad de trigo molido.

Tabla 1. Valores promedios de análisis físico químico y reológico de las variedades ITAPUA 80, ITAPUA 85 y CANINDÉ 21 en tres localidades, Capitán Miranda (CICM), Col. Yguazú (CETAPAR) e Yhovy (CEY) durante los ciclos 2012-14.

\begin{tabular}{|c|c|c|c|c|c|c|c|c|c|}
\hline \multirow[b]{2}{*}{ Parámetros } & \multicolumn{3}{|c|}{ Capitán Miranda (CICM) } & \multicolumn{3}{|c|}{ Col. Yguazú (CETAPAR) } & \multicolumn{3}{|c|}{ Yhovy (CEY) } \\
\hline & $\begin{array}{c}\text { İtapúa } \\
80\end{array}$ & $\begin{array}{l}\text { Ittapúa } \\
85\end{array}$ & $\begin{array}{c}\text { Canindé } \\
21\end{array}$ & $\begin{array}{c}\text { İtapúa } \\
80\end{array}$ & $\begin{array}{c}\text { Itapúa } \\
85\end{array}$ & $\begin{array}{c}\text { Canindé } \\
21\end{array}$ & $\begin{array}{c}\text { İtapúa } \\
80\end{array}$ & $\begin{array}{c}\text { Itapúa } \\
85\end{array}$ & Canindé 21 \\
\hline $\begin{array}{l}\text { Peso hectolítrico } \\
\left(\mathrm{kg} \mathrm{hL}^{-1}\right)\end{array}$ & 78,7 & 76 & 76,3 & 76,2 & 76 & 76,3 & 77,6 & 78,4 & 78,6 \\
\hline $\begin{array}{l}\text { Peso mil granos } \\
(\mathrm{g})\end{array}$ & 29,85 & 32 & 30,5 & 31,65 & 30 & 32 & 33,85 & 33,6 & 31 \\
\hline Proteína (\%) & 13,25 & 13,6 & 13,9 & 12,2 & 13,19 & 13,32 & 15,07 & 12,8 & 14 \\
\hline $\begin{array}{l}\text { Gluten húmedo } \\
(\%)\end{array}$ & 30,2 & 36,3 & 34,75 & 37,3 & 35 & 33,5 & 33,75 & 39,4 & 38,1 \\
\hline $\begin{array}{l}\text { Falling Number } \\
\text { (s) }\end{array}$ & 392 & 326 & 486 & 285 & 363 & 340 & 212 & 374 & 348 \\
\hline $\begin{array}{l}\text { Fuerza del gluten } \\
\mathrm{W}\left(10^{-4} \mathrm{~J}\right)\end{array}$ & 255 & 278 & 314 & 261 & 249 & 273 & 278 & 297 & 369 \\
\hline
\end{tabular}

* La clase comercial del trigo está basado en el valor de la Alveografía (W), considerando los valores entre 180 y 240 como trigo pan, 241 300 como trigo superior y más de 300 como trigo mejorador. Instrução Normativa No 7,15 de agost o de 2001 , do MAA. Norma de Identidade e qualidade do trigo. Diário Oficial da Unión, secao 1. Brasil. 
El porcentaje de proteína es una medición indirecta del contenido de gluten en el grano, pero no de su calidad. Las harinas para pan provienen de trigos que contienen como mínimo $12 \%$ o $13 \%$ de proteína y aquellos con menos del $11 \%$ no son aconsejados para producir pan a menos que se mezcle con otros para lograr la proteína necesaria (Felícitas et al. 2003). Los valores de proteína de las variedades Itapúa 80, Itapúa 85 y Canindé 21 oscilaron entre $12,2 \%$ y $15,07 \%$, considerados como medio $(11,6 \%$ a $13,5 \%)$ a alto $(13,6 \%$ a $15,5 \%)$. Según Islas-Rubio et al. (2005), Calvel (1983) y Tejero (1995) estos valores de proteína permiten a las tres variedades ser aptas para la fabricación de pan francés, pan de forma y para mezclas con harinas débiles (Tabla1).

Como mencionado anteriormente, el valor de gluten húmedo de una variedad está relacionado con su contenido de proteína. Alto contenido del gluten en trigo permite una buena retención de gases que se desprenden durante la fermentación en el proceso de panificación y resultando en altos volúmenes de pan (Gómez et al. 2007). A pesar de la variación observada entre las localidades de prueba, todos los valores de Gluten húmedo de la variedad Itapúa 80 (Tabla 1), se encuentran entre normal (28\% a 30\%) y excelente (37\% a $40 \%)$. Mientras, que en las localidades de Capitán Miranda (CICM) y Col. Yguazú (CETAPAR) la variedad Itapúa 85 presentó valores dentro del rango de bueno (31\% $36 \%$ ) y en la localidad de Yhovy (CEY) dentro del rango de excelente $(37 \%-40 \%)$. En todos los casos estos valores permiten a las variedades la formación de una red tridimensional, lo que se traduce en una masa cohesiva, extensible y con capacidad de retener los gases producidos en la fermentación (León y Rosell 2007). De igual manera, los valores de porcentaje de Gluten húmedo de la variedad Canindé 21 son buenos en las localidades de Capitán Miranda (CICM) y Col. Yguazú (CETAPAR) y excelentes en Yhovy (CEY). Esto se podría atribuir más a su calidad proteica que a su contenido, que se traduce en un buen volumen de pan (Vázquez 2009).

Los valores de Falling Number (FN) o índice de caída, indican la actividad amilolítica responsables de la hidrólisis del almidón y de la producción de maltosa, azúcar que servirá de sustrato para la fermentación y la generación de gas $\left(\mathrm{CO}_{2}\right)$ durante este proceso (Gómez et al. 2007, Magaña et al. 2009). Los valores de FN de las tres variedades Itapúa 80, Itapúa 85 y Canindé 21 se encuentran entre ideal (201 s a 350 s) y de baja (>351 s) actividad enzimática presentadas en la Tabla 1. Las diferencias de valores observadas en las variedades entre diferentes localidades y ciclo del cultivo son meramente causadas por condiciones climáticas durante el llenado del grano y periodo de cosecha. Solo en el caso de la variedad Canindé 21 el valor muy alto de 486 s en Capitán Miranda demostró una baja eficiencia para fermentar (Gómez 2007).

La baja actividad enzimática facilita el almacenamiento del grano y no constituye un problema para la industria panadera, debido a la existencia de reforzadores o mejoradores enzimáticos utilizados en el proceso de la panificación (Calvel 1983, Tejero 1995, Miralbés 2000). También, Seghezzo y Molfese (2006) mencionan que la baja actividad enzimática no representa problema para la industria considerando el uso común de aditivos y enzimas alfa amilasas que permiten la corrección de las harinas en la molinería y en la elaboración de panes o ser aptas para productos no fermentables como son las galletitas (Calvel 1983, Tejero 1995).

Los valores de la fuerza de gluten (W) obtenidos a través del alveógrafo de Chopin nos permite comprobar la adecuación de las distintas harinas a diferentes procesos de panificación y de la elaboración de galletas etc. (Indrani et al. 2007). Además, es uno de los parámetros más importantes ya que permite clasificar a los trigos en duros, semiduros y blandos, de acuerdo a su aptitud de uso industrial (Bettege et al. 1989, Indrani et al. 2007). Los ensayos de alveografía son muy empleados en la industria, ya que sus resultados permiten clasificar a las harinas de trigo en tres grupos principalmente: para panificación, para la elaboración de pastas y para la elaboración de galletitas.

Los valores de $\mathrm{W}$ presentados en la Tabla 1 clasifican a la variedad Itapúa 80 como medio-fuerte (201 a 300 10-4 Joule). Este rango representará mayor capacidad para resistir el trabajo mecánico durante el amasado, retener gases de la fermentación y dar pan de buen volumen y presentación. Estos valores de $\mathrm{W}$ son bien aceptados por la industria nacional ubicando a la variedad Itapúa 80 con un buen potencial de panificación por su obtención de masas equilibradas que desarrollan bien durante la fermentación y cocción (Mesas y Alegre 2002). Aunque los valores de $\mathrm{W}$ de la variedad Itapúa 85 también se encuentran en el rango de medio-fuerte, la estabilidad de estos valores durante años y localidades lo clasifica a la variedad Itapúa 85, como apta para la panificación industrial y para las mezclas de las harinas con trigos de gluten débil.

La variedad Canindé 21 es muy distinta en sus valores del $\mathrm{W}$ y se encuentra en el rango de fuerte (301-400 $10^{-4}$ Joule) en Capitán Miranda (CICM) y en Yhovy (CEY). 
La clasificación del valor de $\mathrm{W}$ considerada medio fuerte para esta variedad en la localidad de Col. Yguazú (CETAPAR) se debió a la deficiente aplicación de fertilizante nitrogenado al suelo y a la planta durante los años en estudio ${ }^{1}$ la que actúa sobre la calidad panadera presentando una disminución en su W (López Bellido 2013).

De acuerdo a sus valores de W, estas variedades no solo se pueden usar para la panificación mecanizada, sino también para mezclas con otros trigos de valor $\mathrm{W}$ bajo o débil para los distintos productos finales. Estos valores de W garantizan el poder de la harina para la elaboración de pan de buena calidad (De la Vega 2009).

Según la Instrucción Normativa $\mathrm{N}^{\circ} 7$ de 2001 del MAA del Brasil, las variedades Itapúa 80 e Itapúa 85 se encuentran dentro de la clase de superior a pan visto por sus valores de fuerza del gluten W. Esta normativa lo clasifica como variedades aptas para la panificación tradicional y mezclas con harinas débiles.

A su vez y en función a los valores de fuerza del gluten W, la variedad Canindé 21 se encuentra dentro de la Clase de Mejorador, apta para panificación mecanizada y mezclas con harinas débiles

\section{CONCLUSIONES}

Las tres nuevas variedades de trigo, Itapúa 80, Itapúa 85 y Canindé 21, presentan calidad industrial de acuerdo a los parámetros de calidad requeridos por el mercado nacional e internacional para la fabricación de pan y de otros productos.

Se destaca la calidad de la variedad Canindé 21 con un alto valor de W (Fuerza del Gluten) que la ubica en la clase Mejorador, siendo apta para panificación mecanizada y para mezclas con harinas débiles.

El productor dispone para elegir entre estas variedades que además de poseer alto potencial de rendimiento y sanidad presentan una calidad diferenciada.

\section{REFERENCIAS BIBLIOGRAFICAS}

AACC (American Association of Cereal Chemists, Estados Unidos de América). 2000. Approved methods of the American Association of Cereal Chemists. St. Paul, Estados Unidos América, AACC.

\footnotetext{
${ }^{1}$ Centurión, F. 22 may. 2013. Aplicación de nitrógeno para fertilización de trigo (entrevista). Colonia Yguazú, Paraguay, Centro Tecnológico Agropecuario del Paraguay - CETAPAR.
}

Bettege, A; Rubenthaler, GL; Pomeranz, Y. 1989. Alveograph algoritthms to predict function-alproperties of wheat in bread and cookie baking. Cereal Chemistry 66:81-86.

Calvel, R. 1983. La panadería moderna. 2 ed. Buenos Aires, Argentina, América Lee. 410 p.

Cuniberti, M; Nisi, J; Masiero, B. 2004. Relación rendimiento vs. calidad industrial: estabilidad en la calidad de variedades de trigo. Cordoba, Argenina, INTA. 28 p. (Serie: Actividades de Difusión No 444)

De la O, OM; Espitia, HE; Villaseñor, M; Molinas, JD; López, S; Santacruz, V; Peña, RJ. 2010. Proteínas del gluten y reología de trigos harineros mexicanos influenciados por factores ambientales y genotipos. Pesq Agropec Bras. 45:989-996.

De la Vega Ruiz, G. 2009. Proteínas de la harina de trigo: clasificación y propiedades funcionales. México. Temas de Ciencias y Tecnología 13(38):27-32.

Felícitas, H; Mellado, M; Wilckens, R; Berti, M; Jofré, S. 2003. Rendimiento de harina y aptitud panadera de seis cultivares de trigo de primavera sembrado en tres ambientes. Agrosur 31(2):38-46.

Gaines, CS; Finney, PL; Andrews, C. 1997. Influence of kernel size and shriveling on soft wheat milling and baking quality. Cereal Chemistry 74(6):700-704.

Gómez Pallarez, M; León, AE; Rosell, CM. 2007. Trigo: productos de panificación. In Leon, AE; Rossell, CM. (eds.). De tales harinas, tales panes: granos, harinas y productos de panificación en Iberoamérica. Córdoba, Argentina, Hugo Báez. p. 52-53.

Halvers on, J; Zeleny, L. 1988. Criteria of wheat quality. In Pomeranz, Y. (ed.). Wheat chemestry and Technology. St. Paul, Estados Unidos de América, AACC. p. 15-24.

Indrani, D; Manohar, RS; Rajiv, J; Rao, GV. 2007. Alveograph as tool asses the quality characteristics of wheat fluor for parotta making. Journal of Engineering 78:1202-1206.

Islas-Rubio, AR; MacRitchie, F; Gandikota, S; Hou, G. 2005. Relaciones de la composición proteínica y mediciones reológicas en masa con la calidad panadera de harinas de trigo. Rev. Fitotec. Mex. 28(3):243-251.

IRAM (Instituto Argentino de Normalización y Certificación, Argentina). 2000. Métodos aprobados por el Instituto Argentino de Normalización y Certificación. Buenos Aires, Argentina, IRAM. s.p. 
López Bellido, L. 2013. Abonado de los cereales trigo y cebada (en línea). Córdoba, España, ETSIA. Consultado 15 julio 2015. Disponible en http://www.tecnicoagricola.es/abonado-de-loscereales-trigo-y-cebada/

MAA (Ministério da Agricultura do Abastecimento, Brasil). Instrução Normativa $N^{o}$ 7, 15 de agosto de 2001, do MAA. Norma de Identidade e qualidade do trigo. Diário Oficial da Unión, secao 1. Consultado el 20 de junio de 2011. Disponible en: http://www.codapar.pr.gov.br/arquivos/File/pdf/trigo. pdf

Magaña-Barajas, E; Ramírez-Wong, B; Platt-Lucero, LC; López-Ahumada, GA; Torres, PI; Sánchez-Machado, DI. 2009. Caracterización visco elástica de masas de variedades de trigos suaves. México, Tecnología, Ciencia, Educación 24(1):12-22.

Matus, I; Vega, A. 2004. Variedades: boletín de Trigo. Quilamapu, Chile, INTA. 25 p.

Matus, I. 2010. Mejoramiento genético para rendimiento y calidad de Trigo Candeal. Chillan, Chile, INIA. Centro de Investigación Quilamapu. p. 1-9

Mesas, JM; Alegre, MT; 2002. El pan y su proceso de elaboración. Ciencia y Tecnología Alimentaria. 3(5):307-313.

Miralbés, C. 2000. Enzimas en panadería. Barcelona, España, Montagud, 166 p.

Palmer, GH. 1989. Cereal science and technology. Aberen, Suiza, AUP. 463 p.

Peña, BRJ; Hernández, EN; Pérez, HP; Villaseñor, MHE; Gómez, VMM; Mendoza, LMA. 2008. Calidad de la cosecha del trigo en México: ciclo otoño-invierno 2006-2007. México, CONASIST-CONATRIGO. 28 p.
Seghezzo, ML; Molfese, ER. 2006. Calidad en Trigo pan. Buenos Aires, Argentina, INTA, 35 p. (Boletín Técnico No 41). ISBN 0327-8735.

Tejero, F. 1995. Panadería española II. Barcelona, España, Montagud, p. 20-31.

Vázquez, D; Watts, B; Townley-Smith, F; Lukow, O. 2001. Influence of polyphenol oxidase activity and grain color on noodle color. In International Wheat Quality Conference (2, 2001, Manhattan, Estados Unidos de América).

Vázquez, D. 2006. Introducción a la calidad: determinantes de la calidad: parámetros y su importancia a nivel industrial. In Jornadas de Cultivos de Inviernos "Calidad vs Rendimiento". Uruguay, INIA, p. 1-3. (Serie Actividades de Difusión No 444)

Vázquez, D. 2009. Aptitud industrial de Trigo. La Estanzuela, Uruguay, INIA. 45 p. (Serie Técnica 177).

Williams, P. 1988. Crop Quality Evaluation Methods and Guidelines. 2 ed. Aleppo, Syria, ICARDIA. 145 p.

Williams, RM; O' Brien, LO; Eagles, HA; Solah, VA; Jayasena, V. 2008. The influence of genotype, enviromment and genotype $\mathrm{x}$ enviromment interation on wheat quality. Aust.J. Agric. Res. 59:95-111.

Wilson, H; Godiño, M. 2000. Tecnología de almacenamiento de granos de trigo. 2 ed. Montevideo, Uruguay, INIA. 123 p. (Serie Técnica N ${ }^{\circ}$ 107). 